\title{
Catheter ablation for atrial fibrillation in hypertrophic cardiomyopathy: a systematic review and meta-analysis
}

\author{
Rui Providencia, ${ }^{1}$ Perry Elliott, ${ }^{1,2}$ Kiran Patel, ${ }^{2}$ Jack McCready, ${ }^{3}$ Girish Babu, ${ }^{1}$ \\ Neil Srinivasan, ${ }^{1,2}$ Kostantinos Bronis, ${ }^{1}$ Nikolaos Papageorgiou, ${ }^{1,2}$ Anthony Chow, \\ Edward Rowland, ${ }^{1}$ Martin Lowe, ${ }^{1}$ Oliver R Segal, ${ }^{1}$ Pier D Lambiase ${ }^{1,2}$
}

\begin{abstract}
- Additional material is published online only. To view please visit the journal online (http://dx.doi.org/10.1136/ heartjnl-2016-309406).

${ }^{1}$ Barts Heart Centre, Barts Health NHS Trust, London, UK ${ }^{2}$ Institute of Cardiovascular Science, University College of London, London, United Kingdom

${ }^{3}$ Cardiology Department, Royal Sussex County Hospital, Brighton and Sussex University Hospitals NHS Trust, Brighton, UK
\end{abstract}

\section{Correspondence to} Dr Pier D Lambiase, Professor of Cardiology, Institute of Cardiovascular Science, University College of London, Barts Heart Centre, Barts Health NHS Trust, West Smithfield, London EC1A 7BE, UK; pierlambiase@hotmail.com

Received 1 February 2016 Revised 15 April 2016 Accepted 19 April 2016 Published Online First 27 May 2016

\section{Linked}

- http://dx.doi.org/10.1136/ heartjnl-2016-309761 CrossMark

To cite: Providencia $R$ Elliott $P$, Patel $K$, et al. Heart 2016:102:1533-1543.

\section{ABSTRACT \\ Objective Atrial fibrillation (AF) is common in} hypertrophic cardiomyopathy (HCM) and is associated with a high risk of stroke. The efficacy and safety of catheter ablation in this setting is poorly characterised. We aimed to systematically review the existing literature and to perform a meta-analysis to determine the efficacy and safety of catheter ablation of AF in patients with HCM.

Methods Random-effects meta-analysis of studies comparing HCM versus non-HCM controls. The outcomes of freedom from AF/atrial tachycardia, and acute procedure-related complications were assessed. Studies were searched on MEDLINE, EMBASE, COCHRANE and clinicaltrials.gov.

Results Fourteen studies were considered eligible for the systematic review, of which five were included in the meta-analysis. Freedom from AF/atrial tachycardia relapse was higher in patients without $\mathrm{HCM}$ (after a single procedure: $38.7 \% \mathrm{HCM}$ vs $49.8 \%$ controls, $\mathrm{OR}=2.25,95 \% \mathrm{Cl} 1.09$ to $4.64, \mathrm{p}=0.03$; after $\geq 1$ procedure: $51.8 \% \mathrm{HCM}$ vs $71.2 \%$ controls, $\mathrm{OR}=2.62$, $95 \% \mathrm{Cl} 1.52$ to $4.51, p=0.0006 ; \mathrm{I}^{2}=33 \%$ and $26 \%$, respectively). Risk of procedure-related adverse events was low. Repeat procedures (mean difference $=0.16$, $95 \% \mathrm{Cl} 0.0$ to $0.32, \mathrm{p}=0.05, \mathrm{I}^{2}=53 \%$ ) and antiarrhythmic drugs $(\mathrm{OR}=4.70,95 \% \mathrm{Cl} 2.31$ to 9.55 , $p<0.0001, I^{2}=0 \%$ ) are more frequently needed in patients with HCM to prevent arrhythmia relapse. Sensitivity analyses suggested that the outcome in patients with $\mathrm{HCM}$ with less dilated atria and paroxysmal AF may be more comparable to the general population.

Conclusions The observed complication rate of catheter ablation of AF in patients with HCM was low. Even though the risk of relapse is twofold higher, catheter ablation can be effective in patients with HCM and $A F$, particularly in patients with paroxysmal $A F$ and smaller atria.

\section{BACKGROUND}

Hypertrophic cardiomyopathy (HCM) is the most frequent monogenic cardiovascular disease affecting 1 out of every 500 individuals in the general population. ${ }^{1}$ Atrial fibrillation (AF) is the most common arrhythmia in patients with HCM with a prevalence and annual incidence $22.5 \%$ and $3.1 \%$, respectively. $^{2} \mathrm{New}$-onset AF is often associated with heart failure symptoms ${ }^{3}$ and requires prompt treatment with direct current cardioversion in haemodynamically unstable patients or ventricular rate control with oral ß-blockers or non-dihydropyridine calcium channel antagonists followed by elective cardioversion. ${ }^{4}$ There are no randomised controlled trials examining the effect of antiarrhythmic drugs on long-term prevention of AF in patients with HCM and the encouraging results in observational studies which took place decades ago ${ }^{5-7}$ are conflicting with our daily practice, as antiarrhythmic agents are frequently ineffective in eradicating arrhythmias. Similarly, studies assessing the impact of catheter ablation of AF in patients with HCM are sparse and provide contradictory results. The joint Heart Rhythm Society/European Heart Rhythm Association/European Cardiac Arrhythmia Society expert consensus statement on catheter ablation suggests that registries could facilitate the collection of more robust information on the safety and efficacy of AF ablation in the setting of less common underlying conditions, such as HCM. ${ }^{8}$

The aim of this study is to systematically review the existing literature and to perform a meta-analysis of observational studies to determine the efficacy and safety of catheter ablation of AF in patients with HCM.

\section{METHODS}

\section{Study selection}

We performed a search in the databases MEDLINE, EMBASE and COCHRANE (from inception to 7 July 2015) using the following search string: 'catheter ablation' AND 'HCM' AND 'AF'. Reference lists of all accessed full-text articles were searched for sources of potentially relevant information. Ongoing studies assessing the outcomes of catheter ablation of $\mathrm{AF}$ in patients with HCM were searched on ClinicalTrials.gov, and experts in the field were contacted to ensure that all important studies had been included. Authors of full-text papers and congress abstract authors were also contacted by email to retrieve additional information.

The population, intervention, comparison and outcome approach was used for conducting the meta-analysis. ${ }^{9}$ The population of interest included patients with HCM and the intervention was catheter ablation of AF. Comparisons were performed 
between HCM and controls (patients without HCM undergoing catheter ablation of $\mathrm{AF}$ ). The outcomes were mid-term procedural success, need of antiarrhythmic drugs after successful ablation, number of catheter ablation procedures and procedural complications.

Procedural success was defined as freedom from AF or atrial tachycardia relapse, with ECG documentation, after a blanking period. Procedural complications included in the analysis were thromboembolic events (including stroke and transient ischaemic attack), pericardial tamponade requiring pericardiocentesis or pericardial effusion causing haemodynamic imbalance and necessitating prolonged monitoring, pulmonary vein (PV) stenosis, atrio-oesophageal fistula and procedure-related death occurring in the first 30 days post procedure.

To meet inclusion criteria, studies were required to provide information on age, gender and AF type (ie, paroxysmal, persistent or permanent).

Studies providing no information regarding follow-up duration, and number of events in each group were excluded. Similarly, studies consisting of catheter ablation of the atrioventricular node or surgical ablation, and conference abstracts not published as full-text articles in the 5 years following presentation were not examined. Studies presenting data in patients with HCM but not in controls were included in the systematic review, but excluded from the meta-analysis.

Search results were reviewed and consensus reached by three investigators (RP, KP and GB) to ensure that all studies met the prespecified inclusion criteria.

Study quality was formally evaluated using a modified Newcastle-Ottawa Quality Assessment Scale for Cohort Studies ${ }^{10}$ by three reviewers (RP, KB and NS). An agreement between these three reviewers was mandatory for the final classification of studies.

\section{Data extraction}

Data extraction and presentation for the preparation of this manuscript followed the recommendations of the Preferred Reporting Items for Systematic Reviews and Meta-Analyses (PRISMA) group. ${ }^{11}$ From each study, we retrieved study design, study population characteristics (age range, gender and AF type, mitral regurgitation, left ventricular outflow tract obstruction, previous myectomy or septal ablation), follow-up duration, lesion set used in the ablation procedure, definition of relapse, postprocedural monitoring, use of antiarrhythmic agents after blanking, predictors of relapse, mid-term outcomes and procedural complications.

\section{Statistical analysis}

Data were pooled using random-effects according to the Mantel-Haenszel model (Review Manager, RevMan, V.5.1. Copenhagen: The Nordic Cochrane Centre, The Cochrane Collaboration, 2011). A random-effects model was chosen for more precisely addressing different effect sizes and non-uniform variation across studies.

Comparisons between patients with HCM and patients without HCM were performed using OR, or mean difference when appropriate, and respective 95\% CIs were shown. Outcomes were maintenance of sinus rhythm after one catheter ablation procedure, or after one or more catheter ablation procedures, number of ablation procedures, need of antiarrhythmic drugs following a successful ablation and procedural complications. Weights of each study in forest plots were calculated using the inverse variance method. Sensitivity analyses were performed excluding data from studies published only as conference abstracts for left atrial size, prevalence of individuals with persistent AF and left atrial size (comparison of studies below vs above median level for the last two scenarios).

Statistical heterogeneity on each outcome of interest was assessed and quantified using the $\mathrm{I}^{2}$ statistic, which describes the percentage of total variation across studies due to heterogeneity rather than chance. Values below 25\%, between $25 \%$ and $50 \%$, and higher than $50 \%$ are, by convention, classified as low, moderate and high degrees of heterogeneity, respectively. ${ }^{12}$

Funnel plots and meta-regression analyses were not performed as part of the assessment for the presence of publication bias, and possible association of baseline differences with modulator variables in procedural outcomes, respectively, as comparisons involved less than 10 studies, which is the minimum number for assuring the appropriateness of these methods. ${ }^{13}$

\section{RESULTS}

\section{Search results}

A total of 209 entries were retrieved for analysis of titles and abstracts. Of these, 177 were excluded as they were either duplicates or deemed unsuitable for the purpose of the meta-analysis (editorials, letters, reviews or case reports). The remaining 32 studies were carefully screened and after analysis of their abstracts and/or full-text only ${ }^{14-27}$ (one was a conference abstract $^{22}$ ) were considered adequate for inclusion in the systematic review (figure 1). Of these, only six studies, ${ }^{16} 1922-25$ provided enough details to be included in the meta-analysis. There was full agreement between investigators (RP, KP and GB) on the inclusion of the selected studies.

\section{Baseline data: patients with HCM undergoing catheter ablation of $\mathrm{AF}$}

The design of selected investigations and baseline data are summarised in tables 1 and 2 . The final population of the systematic review included 403 patients with HCM; 139 patients with HCM and 393 controls were included in the meta-analysis. All included studies were observational and non-randomised, and

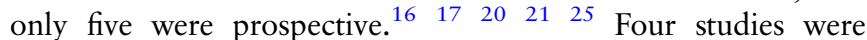
multicentre. $^{14151821}$

Quality assessment of the included studies is shown in table 3. Study quality was modest, with only two studies ${ }^{16}{ }^{23}$ being assigned 7 out of 9 possible points with the Newcastle-Ottawa scale.

The median HCM cohort size was 27 patients (IQR 2239.5). Only one observational study included more than 50 patients with $\mathrm{HCM}^{18}$ In the six studies included in the meta-analysis, treatment groups were balanced for all baseline variables (tables 1 and 2). Diagnosis of HCM was mostly based on the American College of Cardiology Foundation (ACCF) and European Society of Cardiology consensus, ${ }^{34}$ the recent ACCF/ American Heart Association guidelines, ${ }^{29}$ or other preceding documents. $^{28}$ 30-32 One study had genotype information in 11 patients $^{18}$ and one provided no diagnostic criteria for diagnosing HCM. ${ }^{22}$

Median age was 57 years (IQR 54-59). Women accounted for the minority of the patients with HCM, with a median prevalence of 30\% (IQR 26\%-33\%). Persistent AF was the most common AF type in seven studies. ${ }^{19} 202123-26$ The median prevalence of non-paroxysmal AF was 53\% (IQR 37\%-69\%) (table 1).

In studies reporting time since AF diagnosis, ${ }^{14-18} 2021232627$ the median duration was 5.9 years (IQR 4.0-6.9). Median left atrial size was $47 \mathrm{~mm}$ (IQR 46-51 mm) and median maximum left ventricular thickness was $18 \mathrm{~mm}$ (IQR 18-21 mm). Only 
Search on MEDLINE (via PubMED), EMBASE and COCHRANE (from

inception to $9^{\text {th }}$ July 2015 ) using the following search string:

"catheter ablation" AND "atrial fibrillation"

AND "hypertrophic cardiomyopathy"

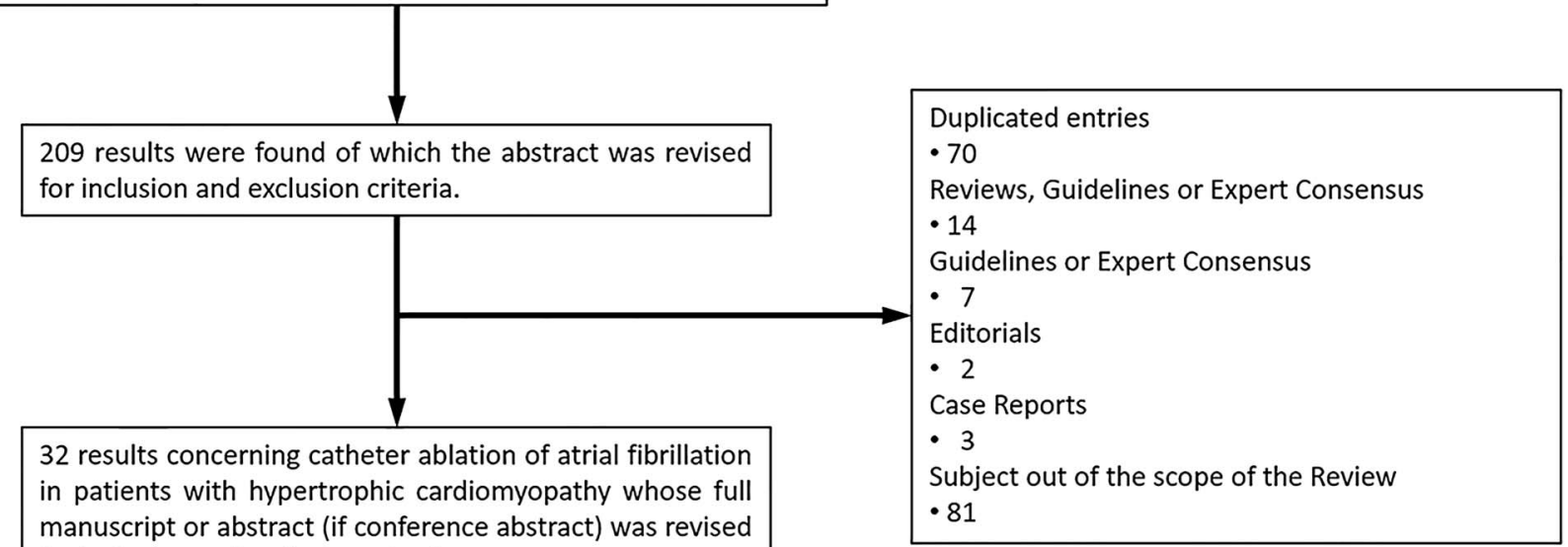

for inclusion and exclusion criteria.

14 studies with information regarding catheter ablation of $\mathrm{AF}$ in 403 patients with $\mathrm{HCM}$.

Figure 1 Study selection diagram. AF, atrial fibrillation; HCM, hypertrophic cardiomyopathy.

nine studies reported on mitral regurgitation, and this was reported as moderate in $7 \%-36 \%$ of patients in six out of the nine studies reporting on this variable. The presence of left ventricular outflow tract obstruction at baseline and previous myectomy or alcohol septal ablation were reported in $11^{14-2023-26}$ and 6 studies, ${ }^{14-16} 1819222426$ respectively, and had a median prevalence of 24\% (IQR 20\%-37.5\%) and 14.5\% (IQR 1.8\%-28.8\%) (table 1).

\section{Procedural data}

All AF ablation procedures consisted of PV isolation and used radiofrequency as the energy source. In two studies, ${ }^{14}{ }^{15}$ the PVs were the only targeted structures, but in the remainder ablation lines were created in the left atrium (LA) and/or right atrium, or lesions deployed to terminate atrial tachycardias (table 2). Ostial PV isolation was performed in two publications, ${ }^{17} 20$ while in the remainder further ablation was performed in a more antral location. In three studies, complex atrial fractionated electrogram ablation was also performed. ${ }^{19-21}$

\section{Procedural outcomes}

The median follow-up was 1.8 years (IQR $1.05-3.30$ years). Except for three studies, ${ }^{14} 1525$ mean follow-up duration was $>1$ year (table 4). In two studies, mean/median follow-up was
$>3$ years. ${ }^{18} 21$ Definition of relapse and monitoring post ablation across all studies are described in table 5.

In four studies, freedom from AF (no documentation of further AF episodes after ablation) at the end of follow-up and after $\geq 1$ procedure was $\geq 70 \% .^{14} 152123$ In two studies, this figure was $60 \%{ }^{16} 18$ and in all remaining studies success rate was lower, in spite of several repeat ablation procedures.

Figure 2 illustrates freedom from arrhythmia in patients with HCM and controls. Control patients had no structural heart disease, except for left ventricular hypertrophy secondary to systemic hypertension in Müssigbrodt et $a l,{ }^{25}$ and in Gaita et al ${ }^{16}$ valvular heart disease was observed in 10 patients and dilated cardiomyopathy in 6 . Both after a single procedure and after $\geq 1$ procedure, sinus rhythm maintenance was lower in patients with HCM: $38.7 \%$ (36/93) HCM vs 49.8\% (148/297) controls, $\mathrm{OR}=2.25,95 \%$ CI 1.09 to $4.64, \mathrm{p}=0.03 ; 51.8 \%(72 / 139)$ HCM vs $71.2 \%$ (280/393) controls, OR $=2.62$, 95\% CI 1.52 to $4.51, p=0.0006$, respectively. Heterogeneity was moderate for both comparisons: $\mathrm{I}^{2}=33 \%$ and $26 \%$, respectively).

The median number of procedures was 1.4 (IQR 1.2-1.5) in patients with HCM and 1.2 (IQR 1.2-1.3) in controls. A second or third ablation procedure was required in $25 \%-50 \%$ of patients with HCM in 10 studies. $^{14} \quad 15 \quad 17-21 \quad 23 \quad 25$ Figure 3 illustrates the comparison of the total number of procedures in 
Table 1 Study design and sample characteristics

\begin{tabular}{|c|c|c|c|c|c|c|c|c|c|c|c|}
\hline Author (year) & $\begin{array}{l}\text { Study } \\
\text { design }\end{array}$ & $\begin{array}{l}\text { Number of HCM and control patients } \\
\text { diagnosis of HCM }\end{array}$ & $\begin{array}{l}\text { Age } \\
\text { (years) }\end{array}$ & q & $\begin{array}{l}\text { Non-paroxysmal } \\
\text { AF }\end{array}$ & $\begin{array}{l}\text { AF } \\
\text { duration } \\
\text { (years) }\end{array}$ & LA size & $\begin{array}{l}\text { LVT } \\
(\mathrm{mm})\end{array}$ & $\begin{array}{l}\% \text { mitral } \\
\text { regurgitation }\end{array}$ & $\begin{array}{l}\text { LVOT } \\
\text { obstruction }\end{array}$ & $\begin{array}{l}\text { Previous } \\
\text { myectomy or } \\
\text { septal ablation }\end{array}$ \\
\hline Liu et al $(2005)^{14}$ & $\begin{array}{l}\text { Retrospective } \\
\text { Multicentre } \\
\text { (two centres) }\end{array}$ & $\begin{array}{l}4 \text { patients with } \mathrm{HOCM} \text { based on } \\
\text { echocardiographic criteria }{ }^{28}\end{array}$ & $58 \pm 8$ & $50 \%(2)$ & $0 \%(0)$ & $8 \pm 8.5$ & $46 \pm 9 \mathrm{~mm}$ & $27 \pm 5$ & N.A. & $100 \%$ (4) & $0 \%(0)$ \\
\hline $\begin{array}{l}\text { Kilicaslan et al } \\
(2006)^{15}\end{array}$ & $\begin{array}{l}\text { Retrospective } \\
\text { Multicentre } \\
\text { (four centres) }\end{array}$ & $\begin{array}{l}27 \text { patients with primary } \mathrm{HCM} \text { according } \\
\text { to } \mathrm{ACCF} / \mathrm{ESC} \text { consensus }{ }^{29}\end{array}$ & $55 \pm 10$ & $30 \%(8)$ & $48 \%(13)$ & $5.4 \pm 3.6$ & $\begin{array}{l}50 \pm 9 \mathrm{~mm} \\
170 \pm 48 \mathrm{~mL}\end{array}$ & $17 \pm 5$ & $\begin{array}{l}\text { Grade } 1-2: 67 \% \\
\text { (18) } \\
\text { Grade 3-4: } 7 \%(2)\end{array}$ & $\begin{array}{l}\text { At rest- } \\
44.4 \%(12) \\
\text { Provoked- } \\
37.0 \%(10)\end{array}$ & $19 \%(5)$ \\
\hline Gaita et al $(2007)^{16}$ & $\begin{array}{l}\text { Prospective } \\
\text { Cohort } \\
\text { Single-centre }\end{array}$ & $\begin{array}{l}26 \text { patients with HCM based on TTE (LV } \\
\geq 13 \text { to } 15 \mathrm{~mm}) \pm \text { family history and } \\
\text { absence of other cardiac or systemic } \\
\text { disease } \\
\text { Controls: } 52 \text { patients }\end{array}$ & $58 \pm 11$ & $31 \%(8)$ & $50 \%(13)$ & $7.3 \pm 6.2$ & $\begin{array}{l}52 \pm 6 \mathrm{~mm} \\
70 \pm 26 \mathrm{~mL} / \mathrm{m}^{2}\end{array}$ & $23 \pm 4$ & $\begin{array}{l}\text { Mild: } 69 \%(18) \\
\text { Moderate: } 12 \%(3)\end{array}$ & $\begin{array}{l}\text { At rest-23\% } \\
\text { (6) }\end{array}$ & $19 \%(5)$ \\
\hline $\begin{array}{l}\text { Bunch et al } \\
(2008)^{17}\end{array}$ & $\begin{array}{l}\text { Prospective } \\
\text { Single-centre }\end{array}$ & $\begin{array}{l}33 \text { patients with HCM } \\
\text { Diagnosis criteria—guidelines/specialised } \\
\text { clinic (Mayo) }\end{array}$ & $51 \pm 11$ & $24 \%(8)$ & $36 \%(12)$ & $6.2 \pm 5.2$ & $\begin{array}{l}51 \pm 7 \mathrm{~mm} \\
140 \mathrm{~mL} \\
(125-180)\end{array}$ & N.A. & $\begin{array}{l}\text { Mild-moderate: } \\
21 \%(7)\end{array}$ & $\begin{array}{l}\text { At rest-24\% } \\
\text { (8) }\end{array}$ & N.A. \\
\hline $\begin{array}{l}\text { Di Donna et al } \\
(2010)^{18}\end{array}$ & $\begin{array}{l}\text { Retrospective } \\
\text { Multicentre } \\
\text { (two centres) }\end{array}$ & $\begin{array}{l}61 \text { patients with HCM based on TTE (LV } \\
\geq 13 \text { to } 15 \mathrm{~mm} \text { ) and absence of other } \\
\text { cardiac or systemic disease } \\
\text { Genotype available in } 11 \text { patients }\end{array}$ & $54 \pm 13$ & $28 \%(17)$ & $43 \%(26)$ & $5.7 \pm 5.5$ & $\begin{array}{l}52 \pm 5 \mathrm{~mm} \\
180 \pm 40 \mathrm{~mL}\end{array}$ & $20 \pm 5$ & $\begin{array}{l}\text { Mild: } 50 \%(28) \\
\text { Moderate: } 36 \%(22)\end{array}$ & $\begin{array}{l}\text { At rest-20\% } \\
(12)\end{array}$ & $10 \%(6)$ \\
\hline $\begin{array}{l}\text { McCready et al } \\
(2011)^{19}\end{array}$ & $\begin{array}{l}\text { Retrospective } \\
\text { Cohort } \\
\text { Single-centre }\end{array}$ & $\begin{array}{l}14 \text { patients with } \mathrm{HCM} \text {-according to } \\
\text { ACCF/ESC consensus }{ }^{29} \\
177 \text { controls }\end{array}$ & $58 \pm 13$ & $21 \%(40)$ & $100 \%$ (191) & N.A. & $47 \pm 7 \mathrm{~mm}$ & $17 \pm 4$ & $\begin{array}{l}\text { Mild: } 14.3 \%(2) \\
\text { Moderate: } 7.1 \% \text { (1) }\end{array}$ & $28.6 \%$ (4) & N.A. \\
\hline $\begin{array}{l}\text { Derejko et al } \\
(2013)^{20}\end{array}$ & $\begin{array}{l}\text { Prospective } \\
\text { observational }\end{array}$ & $\begin{array}{l}30 \text { patients with } \mathrm{HCM} \text { according to } \\
\text { ACCF/ESC consensus }{ }^{29}\end{array}$ & $49 \pm 11$ & $33 \%(10)$ & $53 \%(16)$ & $6 \pm 4.2$ & $51 \pm 7 \mathrm{~mm}$ & $21 \pm 6$ & N.A. & $20 \%(6)$ & $7 \%(2)$ \\
\hline $\begin{array}{l}\text { Santangeli et al } \\
(2013)^{21}\end{array}$ & $\begin{array}{l}\text { Prospective } \\
\text { Multicentre } \\
\text { (eight centres) }\end{array}$ & $\begin{array}{l}43 \text { patients with } \mathrm{HCM} \text { according to } \\
\text { ACCF/ESC consensus }{ }^{29}\end{array}$ & $59 \pm 8$ & $33 \%(14)$ & $72 \%(31)$ & $\begin{array}{l}\text { Median 3.0, } \\
\text { IQR 4.3 }\end{array}$ & $47 \pm 8 \mathrm{~mm}$ & $20 \pm 4$ & N.A. & N.A. & N.A. \\
\hline Yan et al $(2013)^{22}$ & $\begin{array}{l}\text { Retrospective } \\
\text { Cohort } \\
\text { Single-centre }\end{array}$ & $\begin{array}{l}25 \text { patients with HCM } \\
\text { Diagnosis criteria—N.A. } \\
50 \text { controls }\end{array}$ & $\begin{array}{l}53 \pm 8 \\
54 \pm 8\end{array}$ & $\begin{array}{l}24 \%(6) \\
24 \%(12)\end{array}$ & $\begin{array}{l}36 \%(9) \\
40 \%(20)\end{array}$ & N.A. & $47 \pm 8 \mathrm{~mm}$ & N.A. & N.A. & N.A. & N.A. \\
\hline $\begin{array}{l}\text { Hayashi et al } \\
(2014)^{23}\end{array}$ & $\begin{array}{l}\text { Retrospective } \\
\text { Cohort } \\
\text { Single-centre }\end{array}$ & $\begin{array}{l}17 \text { patients with HCM based on TTE (LV } \\
\geq 15 \mathrm{~mm} \text { ) and absence of other cardiac } \\
\text { or systemic disease } 31031 \\
34 \text { controls }\end{array}$ & $\begin{array}{l}63 \pm 12 \\
66 \pm 9\end{array}$ & $29 \%(5)$ & $53 \%(9)$ & $\begin{array}{l}3.5 \pm 3.5 \\
4.1 \pm 3.7\end{array}$ & $46 \pm 7 \mathrm{~mm}$ & $19 \pm 4$ & $\begin{array}{l}\text { Moderate or severe } \\
18 \%(3) \\
9 \%(3)\end{array}$ & $23.5 \%$ (4) & $41 \%(7)$ \\
\hline $\begin{array}{l}\text { Contreras-Valdes } \\
\text { et al }(2015)^{24}\end{array}$ & $\begin{array}{l}\text { Retrospective } \\
\text { Cohort } \\
\text { Single-centre }\end{array}$ & $\begin{array}{l}40 \text { patients with } \mathrm{HCM} \text { according to the } \\
\text { ACCF/AHA guidelines }{ }^{32} \\
64 \text { controls }\end{array}$ & $54 \pm 7$ & $30 \%(12)$ & $\begin{array}{l}68 \%(27) \\
70 \%(45)\end{array}$ & N.A. & N.A. & $18 \pm 3$ & N.A. & $37.5 \%$ (15) & N.A. \\
\hline $\begin{array}{l}\text { Müssigbrodt et al } \\
(2015)^{25}\end{array}$ & $\begin{array}{l}\text { Prospective } \\
\text { Cohort } \\
\text { Single-centre }\end{array}$ & $\begin{array}{l}22 \text { patients with HCM based on TTE (LV } \\
\geq 15 \mathrm{~mm}) \pm \text { LVOT obstruction and } \\
\text { absence of other cardiac or systemic } \\
\text { disease }^{33} \\
22 \text { patients with secondary cardiac } \\
\text { hypertrophy }\end{array}$ & $\begin{array}{l}57 \pm 8 \\
63 \pm 10\end{array}$ & $\begin{array}{l}32 \%(7) \\
36 \%(8)\end{array}$ & $\begin{array}{l}55 \%(12) \\
55 \%(12)\end{array}$ & N.A. & $46 \pm 8 \mathrm{~mm}$ & $19 \pm 4$ & $\begin{array}{l}\text { Significant: } \\
14 \%(3) \\
0 \%(0)\end{array}$ & $36 \%(8)$ & $32 \%(7)$ \\
\hline $\begin{array}{l}\text { Okamatsu et al } \\
(2015)^{26}\end{array}$ & $\begin{array}{l}\text { Retrospective } \\
\text { Single-centre }\end{array}$ & $\begin{array}{l}22 \text { patients with HCM based on the } \\
\text { presence of myocardial hypertrophy and } \\
\text { absence of local or systemic aetiology }\end{array}$ & $65 \pm 11$ & $55 \%(12)$ & $77 \%$ (17) & $6.7 \pm 4.4$ & $\begin{array}{l}48 \pm 6 \mathrm{~mm} \\
98 \pm 38 \mathrm{~mL}\end{array}$ & $13 \pm 4$ & $\begin{array}{l}\text { Greater than or } \\
\text { equal to moderate: } \\
23 \% \text { (5) }\end{array}$ & $14 \%(3)$ & N.A. \\
\hline
\end{tabular}




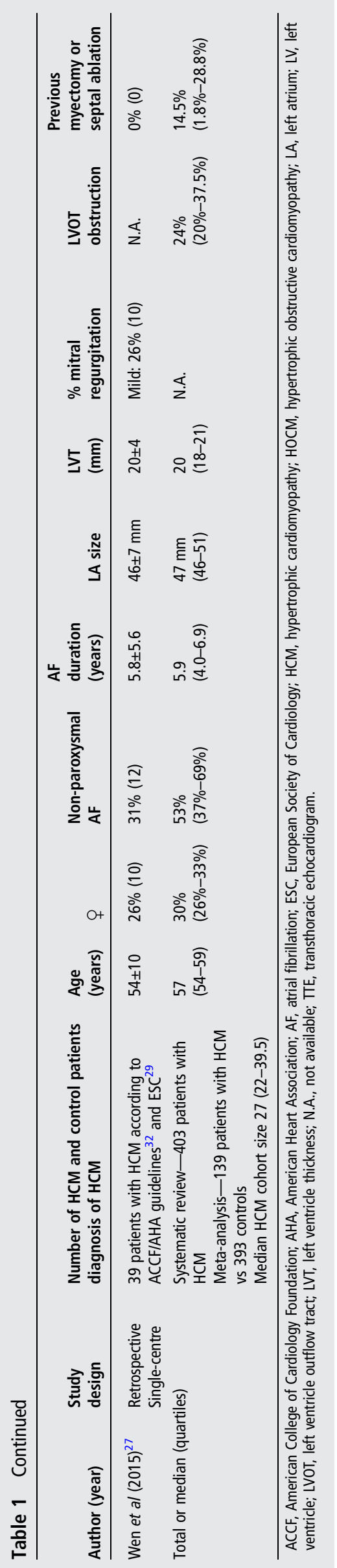

controlled studies, showing that patients with HCM underwent repeat procedures more often: mean difference $=0.16,95 \% \mathrm{CI}$ 0.0 to $0.32, \mathrm{p}=0.05, \mathrm{I}^{2}=53 \%$.

In two studies, patients remained in sinus rhythm free from antiarrhythmic drugs, ${ }^{21}$ or these were used in only a minority of patients. $^{22}$ However, in the remaining studies, antiarrhythmic agents were needed for optimisation of the rhythm control strategy in $>25 \%-50 \%$ of patients with HCM. In controlled studies, chances of remaining on antiarrhythmic drugs following a successful ablation were fivefold higher in patients with HCM: OR=4.70, 95\% CI 2.31 to $9.55, \mathrm{p}<0.0001, \mathrm{I}^{2}=0 \%$ (figure 3). Of note, in some patients with HCM these drugs were used because of concomitant ventricular arrhythmias.

\section{Predictors of procedural success}

Left atrial size was the most frequently identified predictor of procedural success. ${ }^{17} 18{ }^{24-27}$ In two studies, persistent AF was also associated with worse procedural outcomes $(\mathrm{OR}=7.7,95 \%$ CI 1.13 to $50, p=0.02^{20}$ and $\mathrm{OR}=2.58,95 \%$ CI 1.11 to 6.05 , $\left.\mathrm{p}=0.028^{21}\right)$. Other predictors of relapse were identified separately in single studies: age and New York Heart Association (NYHA) class, ${ }^{18}$ left atrial pressure and left ventricle (LV) outflow tract obstruction, ${ }^{24} \mathrm{AF}$ duration in months and $\mathrm{E} / \mathrm{E}^{\prime},{ }^{25}$ and corrected QT interval (QTc) duration ${ }^{27}$ (table 4).

\section{Sensitivity analyses}

Sensitivity analysis after excluding results published as a conference abstract ${ }^{22}$ confirmed that frequency of sinus rhythm maintenance after one or more catheter ablation procedures was twofold higher in patients without HCM: HCM 52.9\% (63/119) versus controls $71.1 \%(248 / 349)$; $\mathrm{OR}=2.52$, 95\% CI 1.28 to 4.93, $\mathrm{p}=0.007, \mathrm{I}^{2}=39 \%$ (see online supplementary figure S1).

Pooling of studies including $\leq 53 \%$ (median $\%$ of persistent $\mathrm{AF}$ ) of subjects with persistent $\mathrm{AF}$ displayed a higher relapse rate in patients with HCM: HCM 61.9\% (39/63) versus controls $76.2 \%(99 / 130) ; \mathrm{OR}=2.05,95 \%$ CI 1.05 to $4.01, \mathrm{p}=0.04$, $\mathrm{I}^{2}=0 \%$. However, data from studies with $>53 \%$ of patients with persistent AF showed an even higher relapse rate in patients with HCM (HCM 43.4\% (33/76) versus controls 76.7\% (181/263); $\mathrm{OR}=3.46,95 \%$ CI 1.22 to $\left.9.78, \mathrm{p}=0.02, \mathrm{I}^{2}=58 \%\right)$, suggesting that persistent $\mathrm{AF}$ is associated with a lack of procedural success (see online supplementary figures S2A and S2B).

Similarly, a sensitivity analysis for left atrial size showed that studies with more severely dilated left atria $(\geq 47 \mathrm{~mm}$, the median LA diameter in the HCM cohort) presented with higher relapse rate in patients with HCM (HCM 45.0\% (27/60) versus controls $64.5 \%(189 / 293)$; OR $=3.52,95 \%$ CI 1.16 to 10.67 , $\mathrm{p}=0.03, \mathrm{I}^{2}=62 \%$ ), whereas pooling of studies with less pronounced degrees of left atrial dilation produced neutral results (HCM 66.7\% $(26 / 39)$ versus controls 78.6\% (44/56); $\mathrm{OR}=1.51,95 \% \mathrm{CI} 0.57$ to $\left.3.98, \mathrm{p}=0.41, \mathrm{I}^{2}=0 \%\right)$, suggesting comparable success rate in patients with HCM to the normal population when the LA is not excessively dilated (see online supplementary figures S3A and S3B).

Funnel plots and meta-regression were not performed, as only six entries were eligible for the meta-analysis.

\section{Complications of AF ablation}

While six studies reported no major complications, thromboembolic complications without permanent sequels occurred in two studies ${ }^{17} 20$ (table 5). PV stenosis was reported in three entries, ranging from $3.0 \%{ }^{17}$ and $4.5 \%{ }^{25}$ to $4.8 \% .^{15}$ Contreras-Valdes et al reported that patients with HCM may have longer postablation hospitalisation and higher readmission 
Table 2 Procedural aspects and use of AADs

\begin{tabular}{|c|c|c|c|}
\hline Author (year) & Ablation procedure & Number of procedures & Use of AADs after blanking \\
\hline Liu et al $(2005)^{14}$ & PVI & $\begin{array}{l}1.3 \\
\text { Second procedure: } 25 \%(1)\end{array}$ & $\begin{array}{l}\text { Oral amiodarone in one patient }(25 \%) \text { to prevent AT } \\
\text { relapses after second procedure }\end{array}$ \\
\hline $\begin{array}{l}\text { Kilicaslan et al } \\
(2006)^{15}\end{array}$ & PVI & $\begin{array}{l}1.3 \\
\text { Second procedure: } 25.9 \%(7)\end{array}$ & $\begin{array}{l}5 \text { of } 13 \text { patients ( } 38.5 \%) \text { with relapse after the first } \\
\text { procedure remained in SR on AADs } \\
1 \text { out of } 2 \text { patients with relapse after the second } \\
\text { procedure remained in SR on AADs }\end{array}$ \\
\hline Gaita et al (2007) $)^{16}$ & $\mathrm{PVI}+$ roof line+mitral isthmus & $\begin{array}{l}1.2 \\
\text { Second procedure: } 19.2 \%(5)\end{array}$ & 10 of 16 patients $(62.5 \%)$ in SR were off AADs \\
\hline Bunch et al (2008) ${ }^{17}$ & $\begin{array}{l}\text { Ostial PVI in } 15 \text { patients+roof line and mitral } \\
\text { isthmus in seven patients } \\
\text { WACA+roof line and mitral isthmus in } 18 \text { patients }\end{array}$ & $\begin{array}{l}1.4 \\
\text { Second procedure: } 39 \%(13)\end{array}$ & $\begin{array}{l}\text { Of the } 78 \% \text { patients in SR at } 1 \text { year, } 14 \% \text { were under } \\
\text { AADs } \\
\text { Of the } 74 \% \text { patients in SR at } 3 \text { years, } 27 \% \text { were under } \\
\text { AADs }\end{array}$ \\
\hline $\begin{array}{l}\text { Di Donna et al } \\
(2010)^{18}\end{array}$ & $\begin{array}{l}\mathrm{PVI}+\text { roof line+mitral isthmus+CTI (under } \\
\text { fluoroscopic guidance in } 15 \text { patients) }\end{array}$ & $\begin{array}{l}1.5 \\
\text { Second procedure: } 52 \%\end{array}$ & $\begin{array}{l}11 \text { of } 17 \text { patients }(64.7 \%) \text { in SR after the first } \\
\text { procedure were on AADs } \\
11 \text { of } 24 \text { patients }(45.8 \%) \text { in SR after the second } \\
\text { procedure were on AADs }\end{array}$ \\
\hline $\begin{array}{l}\text { McCready et al } \\
(2011)^{19}\end{array}$ & $\begin{array}{l}\mathrm{PVI} \pm \text { roof line, mitral isthmus and CFAE ablation at } \\
\text { the discretion of the operator }\end{array}$ & $\begin{array}{l}\text { HCM 1.5; controls } 1.3 \\
\text { Second procedure: } 71.4 \%(10) \\
\text { HCM } \\
\text { Third procedure: } 14.3 \% \text { (2) HCM } \\
\text { Fourth procedure: } 7.1 \%(1) \mathrm{HCM}\end{array}$ & $\begin{array}{l}\text { The two patients with HCM in SR after catheter } \\
\text { ablation were on AADs }\end{array}$ \\
\hline Derejko et al $(2013)^{20}$ & $\begin{array}{l}\text { Ostial } \mathrm{PVI}+\mathrm{CTI} \text { line } \pm \text { mitral isthmus, roof line and } \\
\text { CFAE ablation at the discretion of the operator }\end{array}$ & $\begin{array}{l}1.4 \\
\text { Second procedure: } 43 \%(13)\end{array}$ & $\begin{array}{l}16 \text { patients with no AF/AT relapse at } 12 \text { months were } \\
\text { under } A A D \text { s and these were stopped in five patients }\end{array}$ \\
\hline $\begin{array}{l}\text { Santangeli et al } \\
(2013)^{21}\end{array}$ & $\begin{array}{l}\text { All patients: PVI+posterior wall isolation between } \\
\text { PVs+SVC isolation } \\
\text { Persistent AF: +all posterior wall (CS and left side } \\
\text { of septum)+CFAE (LA and CS) } \\
\text { Redo: +non-PV triggers }\end{array}$ & $\begin{array}{l}1.6 \pm 0.7 \\
\text { Second procedure: } 58 \%(25) \\
\text { (All patients with recurrence) }\end{array}$ & $\begin{array}{l}91 \% \text { of patients in SR at } 12 \text { months, but only } 76 \% \text { off } \\
\text { ADDs }\end{array}$ \\
\hline Yan et al $(2013)^{22}$ & $\mathrm{PVI} \pm$ roof line, mitral isthmus or $\mathrm{CTI}$ line & 1.1 & $\begin{array}{l}\text { Eight of nine patients with } \mathrm{HCM}(88.9 \%) \text { were free } \\
\text { from AF recurrence without AADs }\end{array}$ \\
\hline Hayashi et al $(2014)^{23}$ & $\begin{array}{l}\mathrm{PVI}+\text { roof line+posterior inferior line }+\mathrm{CTI} \pm \text { mitral } \\
\text { isthmus, if persistent } \mathrm{AF}\end{array}$ & $\begin{array}{l}\text { HCM 1.5; controls } 1.4 \\
\text { Second procedure: } 47 \% \text { (8) HCM, } \\
35 \% \text { (12) controls }(p=0.87)\end{array}$ & $\begin{array}{l}\text { AADs used more frequently in patients with } \mathrm{HCM} \\
(47 \% \text { vs } 12 \%, p=0.008)\end{array}$ \\
\hline $\begin{array}{l}\text { Contreras-Valdes et al } \\
(2015)^{24}\end{array}$ & $\begin{array}{l}\text { PVI } \\
\text { Ablation of sustained organised AT }\end{array}$ & $\begin{array}{l}\text { HCM } 1.3 \pm 0.5 \\
\text { Controls } 1.2 \pm 0.4(p=0.7)\end{array}$ & $\begin{array}{l}\text { Chronic AADs in } 45 \% \text { HCM vs } 18.8 \% \text { controls } \\
(p=0.007)\end{array}$ \\
\hline $\begin{array}{l}\text { Müssigbrodt et al } \\
(2015)^{25}\end{array}$ & $\mathrm{PVI} \pm$ roof line, septal line and CTI line & $\begin{array}{l}\text { HCM } 1.4 \text {, controls } 1.1 \\
\text { Second procedure: five patients } \\
\text { with HCM vs three controls } \\
\text { Third procedure: three patients } \\
\text { with HCM }(p=0.045)\end{array}$ & $\begin{array}{l}6 \text { of } 22(27 \%) \text { patients with HCM treated with AADs } \\
\text { vs none in non-HCM group }(p=0.008)\end{array}$ \\
\hline $\begin{array}{l}\text { Okamatsu et al } \\
(2015)^{26}\end{array}$ & $\mathrm{PVI} \pm \mathrm{CTI}$ & $\begin{array}{l}1.1 \\
\text { Second procedure: three patients } \\
\text { with HCM }\end{array}$ & $15(68 \%)$ patients used concomitant AADs \\
\hline Wen et al $(2015)^{27}$ & $\begin{array}{l}\text { Paroxysmal AF: PVI+CTI (if documentation of typical } \\
\text { flutter). } \\
\text { Persistent AF: +roof line, mitral isthmus and CTI }\end{array}$ & 1.0 & N.A. \\
\hline
\end{tabular}

AADs, antiarrhythmic drugs; $A F$, atrial fibrillation; $A T$, atrial tachycardia; $C F A E$, complex fractionated atrial electrograms; $C S$, coronary sinus; $C T I$, cavotricuspid isthmus; $H C M$, hypertrophic cardiomyopathy; LA, left atrium; N.A., not available; PVI, to be interpreted as wide antral circumferential ablation, unless stated ostial PVI; PV, pulmonary vein; SVC, superior vena cava; SR, sinus rhythm; WACA, wide antral circumferential ablation.

rate at 30 days, at the expense of heart failure and congestive symptoms. ${ }^{24}$

Due to the low incidence of major complications, no forest plots could be created as no comparisons were possible between patients with HCM and controls.

\section{DISCUSSION}

This systematic review demonstrates that the success rate of $\mathrm{AF}$ ablation is lower in patients with HCM than in patients without HCM with an overall efficacy of AF ablation in HCM at least 50\% lower than in controls for $\geq 1$ procedure. The need for repeat procedures and maintenance of antiarrhythmic drugs is frequent. Left atrial size and AF type were the most frequently identified
Table 3 Study classification: Newcastle-Ottawa scale for cohort studies

\begin{tabular}{ll}
\hline Article (year) & $\begin{array}{l}\text { Newcastle-Ottawa } \\
\text { Quality Assessment* }\end{array}$ \\
\hline Gaita et al $(2007)^{16}$ & 7 \\
McCready et al $(2011)^{19}$ & 6 \\
Yan et al $(2013)^{24}$ & 6 \\
Hayashi et al (2014) & 7 \\
${\text { Contreras-Valdes et al }(2015)^{24}}_{\text {Müssigbrodt et al }(2015)^{25}}$ & 5 \\
\hline *From 0 to 9 points. & 5 \\
\hline
\end{tabular}


Table 4 Mid-term procedural results and predictors of procedural failure

\begin{tabular}{|c|c|c|c|}
\hline Author (year) & $\begin{array}{l}\text { FUP duration } \\
\text { (years) } \\
\text { Mean } \pm \text { SD or } \\
\text { median (IQR) }\end{array}$ & Predictors of relapse & Mid-term procedural results \\
\hline $\begin{array}{l}\text { Kilicaslan et al } \\
(2006)^{15}\end{array}$ & $0.9 \pm 0.6$ & N.A. & $\begin{array}{l}52 \%(14 / 27) \text { remained in SR after the first procedure; after } \\
\geq 1 \text { procedure this rose to } 70 \%(19 / 27)\end{array}$ \\
\hline Gaita et al $(2007)^{16}$ & $1.6 \pm 0.8$ & N.A. & $\begin{array}{l}58 \%(15 / 26) \text { of patients with } \mathrm{HCM} \text { remained in SR after the } \\
\text { first procedure; this rose to } 62 \%(16 / 26) \text { after } \geq 1 \text { procedure } \\
\text { vs } 65 \%(17 / 26) \text { of patients with secondary LVH and } 77 \% \\
\text { (20/26) with idiopathic AF }\end{array}$ \\
\hline Bunch et al $(2008)^{17}$ & $1.5 \pm 1.2$ & Uni: LA dilation & $\begin{array}{l}\text { Maintenance of SR free from AADs was } 64 \% \text { ( } 95 \% \text { CI } 58 \% \\
\text { to } 72 \%) \text { at } 1 \text { year and } 47 \%(36 \% \text { to } 58 \% \text { ) at } 3 \text { years }\end{array}$ \\
\hline $\begin{array}{l}\text { Di Donna et al } \\
(2010)^{18}\end{array}$ & $\begin{array}{l}\text { Total FUP: } 3.3 \pm 0.7 \\
\text { Post last procedure: } \\
2.4 \pm 1.3\end{array}$ & 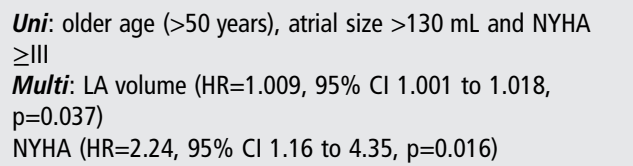 & $67 \%(41 / 61)$ were in SR following $\geq 1$ procedure \\
\hline $\begin{array}{l}\text { Derejko et al } \\
(2013)^{20}\end{array}$ & $1.9 \pm 1.2$ & $\begin{array}{l}\text { Uni: non-paroxysmal AF } \\
\text { Multi: non-paroxysmal (OR=7.7, } 95 \% \mathrm{Cl} 1.13 \text { to } 50, \mathrm{p}=0.02)\end{array}$ & $\begin{array}{l}\text { First procedure success rate was } 33 \%(10 / 30) \text {, and increased } \\
\text { to } 53 \%(16 / 30) \text { after } \geq 1 \text { procedure }\end{array}$ \\
\hline $\begin{array}{l}\text { Santangeli et al } \\
(2013)^{21}\end{array}$ & $\begin{array}{l}3.5(3.2-4.0) \\
\text { Post last procedure: } \\
1.3(0.7-1.6)\end{array}$ & $\begin{array}{l}\text { Uni: long-standing persistent } \mathrm{AF}(\mathrm{OR}=2.58,95 \% \mathrm{Cl} 1.11 \text { to } \\
6.05, \mathrm{p}=0.028)\end{array}$ & $\begin{array}{l}\text { Long-term success rate after a single procedure was } 49 \% \\
\text { and after } \geq 1 \text { procedure } 94 \%\end{array}$ \\
\hline Yan et al $(2013)^{22}$ & $3.3 \pm 1.2$ & N.A. & $\begin{array}{l}\text { SR in } 45 \%(9 / 20) \text { HCM vs } 72 \%(32 / 44) \text { controls after } \geq 1 \\
\text { procedure }(p=0.032)\end{array}$ \\
\hline $\begin{array}{l}\text { Hayashi et al } \\
(2014)^{23}\end{array}$ & $2.2 \pm 1.2$ & N.A. & $\begin{array}{l}\text { SR in } 53 \%(9 / 17) \text { HCM vs } 56 \%(19 / 34) \text { controls after one } \\
\text { procedure (log rank } \mathrm{p}=0.78) \text { and SR in } 82 \%(14 / 17) \mathrm{HCM} \text { vs } \\
88 \%(30 / 34) \text { controls after } \geq 1 \text { procedure (log rank } \mathrm{p}=0.35)\end{array}$ \\
\hline $\begin{array}{l}\text { Contreras-Valdes } \\
\text { et al }(2015)^{24}\end{array}$ & $\begin{array}{l}\text { Median: } 4.5 \\
\text { HCM 1.8-2.3 } \\
\text { Controls 2.9-5.6 }\end{array}$ & $\begin{array}{l}\text { Uni: } \mathrm{LA} \text { pressure } \geq 12 \mathrm{~mm} \mathrm{Hg}(\mathrm{HR}=3.1,95 \% \mathrm{Cl} 1.4 \text { to } 7.1 \text {, } \\
\mathrm{p}=0.005) \text { and dilated } \mathrm{LA}(\mathrm{HR}=1.06,95 \% 1.003 \text { to } 1.11 \text { per } \\
\mathrm{mm} ; \mathrm{p}=0.04) \\
\text { Multi: LVOT obstruction (HR=4.3, } 95 \% \mathrm{Cl} 1.6 \text { to } 11.4 \\
\mathrm{p}=0.0007)\end{array}$ & $\begin{array}{l}42.5 \% \text { HCM vs } 70.3 \% \text { controls remained in SR at } 1 \text { year } \\
\text { after a single procedure ( } p=0.005) \text {; after a redo procedure } \\
\text { this changed to } 45 \% \text { HCM vs } 75 \% \text { controls ( } p=0.001) \\
\text { At the end of FUP } 35 \% \text { of HCM vs } 67.2 \% \text { of controls } \\
\text { ( } p=0.001 \text { ) remained in SR after a single procedure; after a } \\
\text { redo procedure this increased to } 47.5 \% \text { vs } 73.4 \% \text { ( } p=0.005 \text { ) }\end{array}$ \\
\hline Wen et al $(2015)^{27}$ & Mean: 1.2 & $\begin{array}{l}\text { Uni: LA diameter, QTc } \\
\text { Multi: LA diameter (HR=1.072, } 95 \% \mathrm{Cl} 1.004 \text { to } 1.145 \\
\mathrm{p}=0.038) \text {, longer QTC (HR=1.02, } 1.004 \text { to } 1.036, \mathrm{p}=0.013 \text { ); } \\
\text { every } 10 \text { min (HR } 1.227,95 \% \mathrm{Cl} 1.053 \text { to } 1.431, \mathrm{p}=0.009 \text { ) }\end{array}$ & $41 \%(16 / 39)$ remained in SR \\
\hline \multicolumn{4}{|c|}{$\begin{array}{l}\text { AADs, antiarrhythmic drugs; AF, atrial fibrillation; E/E', The ratio of transmitral Doppler early filling velocity to tissue Doppler early diastolic mitral annular velocity; FUP, follow-up; HCM, } \\
\text { hypertrophic cardiomyopathy; LA, left atrium; LVH, left ventricular hypertrophy; LVOT, left ventricle outflow tract; Multi, multivariate analysis; N.A., not available; NS, non-significant; } \\
\text { SD, standard deviation; SR, sinus rhythm; Uni, univariate analysis. }\end{array}$} \\
\hline \multicolumn{4}{|c|}{$\begin{array}{l}\text { predictors of procedural success. Patients with HCM also under- } \\
\begin{array}{l}\text { Given these major factors limiting success, it is remarkable } \\
\text { went ablation late in the course of their disease (median of } \\
5.9 \text { years after the diagnosis of atrial arrhythmias) with non- }\end{array} \text { These data would of } 1.4 \text { procedures, the success rate is } 52 \% \text {. } \\
\text { paroxysmal AF being present in at least } 50 \% \text { of patients in half of } \\
\text { natural history of the disease before it becomes established, then } \\
\text { the included studies. Therefore, by the time of the first procedure the success rates may be higher but this has to be balanced } \\
\text { patients were likely to have a greater degree of electrophysiological } \\
\text { and structural remodelling which further increases the chances of } \\
\text { failure. Furthermore, a significant proportion had mitral regurgita- } \\
\text { and degree of mitral regurgitation and LV diastolic dysfunction } \\
\text { tion and left ventricular outflow obstruction, promoting atrial the likelihood of at least medium-term success. Indeed } \\
\text { stretching, which can shorten the effective atrial refractory period, }\end{array}$} \\
\hline
\end{tabular}


Table 5 Complications and monitoring

Monitoring for AF/AT relapse

Procedural-related complications

\begin{tabular}{|c|c|}
\hline Author (year) & Definition of relapse \\
\hline Liu et al (2005) ${ }^{14}$ & $\begin{array}{l}\text { Any episode of } \mathrm{AF} \text {, regardless of } \\
\text { duration, was considered as arrhythmia } \\
\text { recurrence. }\end{array}$ \\
\hline $\begin{array}{l}\text { Kilicaslan et al } \\
(2006)^{15}\end{array}$ & $\begin{array}{l}\text { Recurrences were based on patient } \\
\text { reporting and rhythm transmitter, Holter } \\
\text { and/or ECG data. }\end{array}$ \\
\hline Gaita et al (2007) ${ }^{16}$ & $\begin{array}{l}\text { Any documented recurrence of AF based } \\
\text { on ECG recordings after } 4 \text { weeks of } \\
\text { blanking. }\end{array}$ \\
\hline $\begin{array}{l}\text { Bunch et al } \\
(2008)^{17}\end{array}$ & $\begin{array}{l}\text { AF elimination if no documented AF } \\
\text { episodes in the absence of AADs. AF } \\
\text { control if remaining in SR without } \\
\text { relapse while on AADs. }\end{array}$ \\
\hline $\begin{array}{l}\text { Di Donna et al } \\
(2010)^{18}\end{array}$ & $\begin{array}{l}\text { Recurrence of } A F \text {, AT or atrial flutter } \\
\text { lasting }>3 \text { min. }\end{array}$ \\
\hline
\end{tabular}

ECG, $24 \mathrm{~h}$ Holter and echocardiography 1, 3, 6 and

9 months after ablation. Monthly telephone interviews. Three patients had a telemetric ECG recorder for 6 months. Two patients had device interrogation.

Outpatient clinic at 3, 6, 12 months and 6 months thereafter. Rhythm transmitter used in the first 3 months (extra 3 months if early recurrence). Forty-eight-hour Holter recording at 3, 6 and 12 months.

Clinical evaluation, 12-lead ECG, echocardiogram and $24 \mathrm{~h}$ Holter monitor at 1, 3, 6, 12 months and every 6 months thereafter.

Telephone contact, clinic follow-up visits and/or communication with referring physician. ECGs and $24 \mathrm{~h}$ Holter in subsequent clinical visits.

Patients followed at 1, 3, 6 and 12 months with ECG, echocardiography and $24 \mathrm{~h}$ Holter and every

6 months thereafter through telephone contact, clinic follow-up visits and communication with the referring physician.

McCready et al Episode of AF or AT $>30 \mathrm{~s}$ documented $(2011)^{19} \quad$ on Holter monitoring or any 12-lead ECG documentation after initial 3 months blanking (on or off AADs).

Derejko et al Recurrence of $A F$, atrial flutter or AT $(2013)^{20} \quad$ lasting $>3$ min, after the initial 3 months documented on ECG or EGM.

Santangeli et al Any episode of AF/AT lasting for $\geq 30 \mathrm{~s}$ $(2013)^{21} \quad$ after initial 3 months blanking.

Yan et al $(2013)^{22} \quad$ N.A

Hayashi et al Episode of AF or AT lasting for $>30 \mathrm{~s}$

$(2014)^{23} \quad$ after the 3-month blanking period.

Contreras-Valdes et al $(2015)^{24}$

Recurrent arrhythmia (AF or AT) after initial 3-month blanking. 12-lead ECGs, Holter monitoring for 1-7 days and pacemaker/implantable cardioverter-defibrillator interrogation (where available).

Clinical appointment, ECG and Holter at 4 weeks and then every 3-6 months. Eight patients underwent 2 weeks of continuous ECG monitoring.

Physical examination, ECG and 7-day Holter monitoring at 3, 6, 9 and 12 months. Event recorder in the first 5 months.

N.A.

Outpatient clinic with ECG every month for the first 12 months and every 2-3 months thereafter. Cardiac event recorder used twice a day for $30 \mathrm{~s} \times 2$ during the first 4 months. A $24 \mathrm{~h}$ Holter monitor 3 months after the procedure and every 12 months thereafter. N.A.

7-day Holter recordings during 6-month, 12-month and 24-month follow-up visits. Interrogation of implantable cardiac devices.

Clinical review, ECG and $24 \mathrm{~h}$ Holter every 13 months.

ECG, $24 \mathrm{~h}$ Holter at 1, 3, 6 and 12 months and every 6 months thereafter. Phone interviews.
Major: none.

Major: asymptomatic PV stenosis: $<50 \%$ in two patients $(7 \%)$ and $50 \%-69 \%$ in two patients (7\%).

Major: none.

Mild pericardial effusion in five patients $(21.7 \%)$.

Major: two patients had a periprocedural TIA and one developed a symptomatic PV stenosis.

\section{Major: none.}

Five $(8 \%)$ patients developed mild

non-haemodynamic comprising pericardial effusion.

Major: cardiac tamponade in one patient $(7.1 \%)$

Major: stroke resolving without sequel after a redo procedure.

Major: none.

Major: none.

Major: none.

\section{Complications: rare.}

Median hospitalisation was longer in HCM 2 (1$6)$ vs $1(1-3), p<0.0001$. Longer readmission rate at 30 days in $\mathrm{HCM} 25 \%$ vs $1.6 \%, \mathrm{p}<0.0003$ (HF and congestive symptoms).

Major: PV stenosis requiring balloon dilation in one patient with HCM.

N.A.

N.A.
$(2015)^{26} \quad$ following a 2-month blanking period.

Wen et al (2015) $27 \quad$ Episode of documented atrial tachyarrhythmia lasting at least $30 \mathrm{~s}$ after a 3-month blanking period.

AADs, antiarrhythmic drugs; AF, atrial fibrillation; AT, atrial tachycardia; EGM, intracavitary electrograms; HCM, hypertrophic cardiomyopathy; HF, heart failure; N.A., not available;

$\mathrm{PV}$, pulmonary vein; SR, sinus rhythm; TIA, transient ischaemic attack.

A number of structural and mechanistic factors further impact on the success rates of AF ablation in HCM. Patients with HCM have a high prevalence of atrial fibrosis, which may serve as a substrate for slow conduction and intra-atrial re-entry, thereby playing a crucial role in the development and maintenance of $\mathrm{AF}^{36}{ }^{37}$ Sarcomeric gene mutations account for $60 \%$ of HCM cases. The $\beta$-myosin heavy chain (MHC) missense mutation Arg663His has been associated with an increased risk of AF in patients with HCM with $47 \%$ Arg663His carriers developing AF over a 7-year follow-up period. ${ }^{38}$ Polymorphisms in the angiotensin receptor gene have also been implicated in the development of AF in HCM. ${ }^{39}$ Anatomical variations in left atrial thickness have been suggested. ${ }^{24}$ However, preliminary data from Hayashi et $a l^{23}$ using CT to measure left atrial thickness in a small sample of patients indicate that left atrial wall in HCM is no thicker than in matched patients without structural heart disease.

Abnormal calcium handling is a recognised pathophysiological mechanism in HCM and could account for triggered activity (from delayed after depolarisations) precipitating AF in the proarrhythmic myocardial tissue architecture. ${ }^{40}$ 


\section{Freedom from AF/AT relapse after a single procedure}

\begin{tabular}{|c|c|c|c|c|c|c|c|c|}
\hline Study or Subgroup & \multicolumn{2}{|c|}{ Control } & \multicolumn{2}{|c|}{$\mathrm{HCM}$} & \multicolumn{2}{|r|}{ Odds Ratio } & \multicolumn{2}{|c|}{$\begin{array}{c}\text { Odds Ratio } \\
\mathrm{M}-\mathrm{H}, \text { Random, } 95 \% \mathrm{Cl}\end{array}$} \\
\hline McCready 2011 & 73 & 177 & 1 & 14 & $10.7 \%$ & $9.13[1.17,71.30]$ & & \\
\hline Hayashi 2014 & 19 & 34 & 9 & 17 & $25.7 \%$ & $1.13[0.35,3.62]$ & & \\
\hline Contreras Valdes 2015 & 45 & 64 & 17 & 40 & $38.5 \%$ & $3.20[1.40,7.31]$ & & $\rightarrow-$ \\
\hline Müssigbrodt 2015 & 11 & 22 & 9 & 22 & $25.0 \%$ & $1.44[0.44,4.76]$ & & \\
\hline Total $(95 \% \mathrm{Cl})$ & & 297 & & 93 & $100.0 \%$ & $2.25[1.09,4.64]$ & & \\
\hline Total events & 148 & & 36 & & & & & \\
\hline $\begin{array}{l}\text { Heterogeneity: } \operatorname{Tau}^{2}=0 \text {. } \\
\text { Test for overall effect: } Z\end{array}$ & $\begin{array}{l}\text { 8; } \mathrm{Chi}^{2}=4 \\
2.18(P=\end{array}$ & $\begin{array}{l}4.45, d t \\
0.03)\end{array}$ & $=3(\mathrm{P}=$ & $0.22) ; 1$ & $=33 \%$ & & $\begin{array}{l}0.010 .1 \\
\text { Controls worse }\end{array}$ & $\begin{array}{c}10 \\
100 \\
\text { HCM worse }\end{array}$ \\
\hline
\end{tabular}

\section{Freedom from AF/AT relapse after one or more procedures}

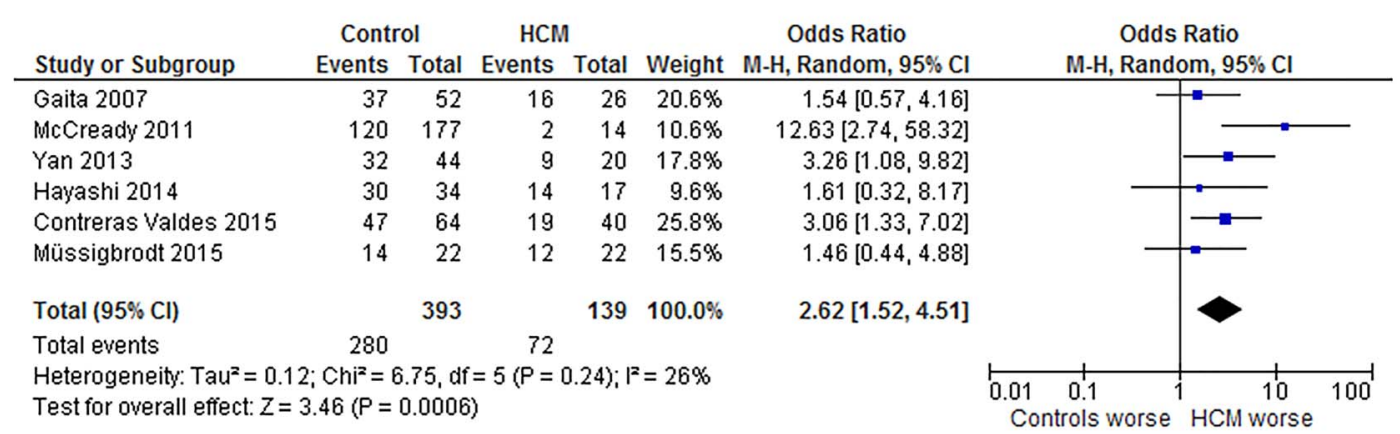

Figure 2 Forest plots comparing procedural outcomes (freedom from AF/AT relapse) of catheter ablation of AF in patients with and without HCM. $A F$, atrial fibrillation; $A T$, atrial tachycardia; HCM, hypertrophic cardiomyopathy.

Myocardial ischaemia ${ }^{41}$ and autonomic dysfunction ${ }^{42}$ are two other factors that have been previously suggested as relevant triggers of $\mathrm{AF}$, and may make $\mathrm{AF}$ ablation more difficult in the context of HCM.
Clearly, understanding the pathophysiology of AF in HCM and identifying predictors of relapse remain important to improve overall procedural outcomes. Santangeli et $a l^{21}$ have suggested that these patients present with frequent non-PV

\section{Number of catheter ablation procedures}

\begin{tabular}{|c|c|c|c|c|c|c|c|c|c|}
\hline \multirow[b]{2}{*}{ Study or Subgroup } & \multicolumn{3}{|c|}{$\mathrm{HCM}$} & \multicolumn{3}{|c|}{ Control } & \multicolumn{2}{|r|}{ Mean Difference } & \multirow{2}{*}{$\begin{array}{l}\text { Mean Difference } \\
\text { IV, Random, } 95 \% \mathrm{Cl}\end{array}$} \\
\hline & Mean & SD & Total & Mean & SD & Total & Weight & IV, Random, $95 \% \mathrm{Cl}$ & \\
\hline Gaita 2007 & 1.19 & 0.4 & 52 & 1.23 & 0.4 & 26 & $25.0 \%$ & $-0.04[-0.23,0.15]$ & \\
\hline McCready 2011 & 1.86 & 0.5 & 14 & 1.41 & 0.6 & 177 & $17.8 \%$ & $0.45[0.17,0.73]$ & \\
\hline Hayashi 2014 & 1.47 & 0.5 & 17 & 1.35 & 0.5 & 34 & $16.8 \%$ & $0.12[-0.17,0.41]$ & \\
\hline Contreras Valdes 2015 & 1.32 & 0.5 & 40 & 1.18 & 0.4 & 64 & $25.4 \%$ & $0.14[-0.04,0.32]$ & \\
\hline Müssigbrodt 2015 & 1.36 & 0.7 & 22 & 1.14 & 0.3 & 22 & $15.1 \%$ & $0.22[-0.10,0.54]$ & \\
\hline Total $(95 \% \mathrm{Cl})$ & & & 145 & & & 323 & $100.0 \%$ & $0.16[0.00,0.32]$ & \\
\hline \multicolumn{9}{|c|}{$\begin{array}{l}\text { Heterogeneity: } \text { Tau }^{2}=0.02 ; C h i^{2}=8.59, d f=4(P=0.07) ; I^{2}=53 \% \\
\text { Test for overall effect: } Z=1.96(P=0.05)\end{array}$} & $\begin{array}{ccc}-0.5-0.25 & 0 & 0.25 \\
\text { Controls more } & 0 \\
\text { HCM more }\end{array}$ \\
\hline
\end{tabular}

\section{Need for AADs in patients with successful ablation}

\begin{tabular}{|c|c|c|c|c|c|c|c|c|c|}
\hline Study or Subgroup & \multicolumn{2}{|c|}{$\mathrm{HCM}$} & \multicolumn{2}{|c|}{ Control } & Weight & $\begin{array}{c}\text { Odds Ratio } \\
\text { M-H, Random, } 95 \% \mathrm{Cl}\end{array}$ & \multicolumn{3}{|c|}{$\begin{array}{c}\text { Odds Ratio } \\
\text { M-H, Random, } 95 \% \mathrm{Cl}\end{array}$} \\
\hline Hayashi 2014 & 8 & 17 & 4 & 34 & $25.2 \%$ & $6.67[1.62,2\} .38]$ & & & \\
\hline Contreras Valdes 2015 & 18 & 40 & 12 & 60 & $63.7 \%$ & $3.27[1.35,7.95]$ & & & \\
\hline Müssigbrodt 2015 & 6 & 22 & 0 & 22 & $5.8 \%$ & $17.73[0.93,337.26]$ & & & \\
\hline McCready 2011 & 2 & 2 & 28 & 120 & $5.3 \%$ & $16.23[0.76,347.93]$ & & & \\
\hline Total $(95 \% \mathrm{Cl})$ & & 81 & & 236 & $100.0 \%$ & $4.70[2.31,9.55]$ & & & \\
\hline Total events & 34 & & 44 & & & & & & \\
\hline $\begin{array}{l}\text { Heterogeneity: } \operatorname{Tau}^{2}=0 . \\
\text { Test for overall effect: } Z=\end{array}$ & $\begin{array}{l}\mathrm{Chi}^{2}=2 \\
28(\mathrm{P}<\end{array}$ & $\begin{array}{l}.32, \mathrm{df} \\
0.0001\end{array}$ & $=3(P=$ & $0.51) ; I^{2}$ & $=0 \%$ & & $\begin{array}{cc}0.05 & 0.2 \\
\text { Controls more }\end{array}$ & $\begin{array}{c}5 \\
5 \\
\mathrm{HCM} \text { more }\end{array}$ & 20 \\
\hline
\end{tabular}

Figure 3 Forest plots comparing number of ablation procedures (upper panel) and need of AADs following a successful ablation procedure in patients with HCM versus controls. AADs, antiarrhythmic drugs; HCM, hypertrophic cardiomyopathy. 
triggers, which may be responsible for late recurrences. These authors have favoured extensive ablation beyond PV isolation. However, as we demonstrate in this review a consensus on the optimal approach for AF ablation in patients with HCM remains elusive. It is unclear if performing PV isolation and targeting sustained atrial tachycardias is superior to employing a more aggressive approach with extensive lesion sets including lines, targeting complex fractionated electrograms and non-PV triggers in both atria. This is particularly important as even the optimal strategy to identify these sites and their relevance in procedural outcomes is contentious. ${ }^{43}$ Furthermore, the optimal energy source to use is also not clear as all studies in this review have been performed using radiofrequency ablation. A randomised controlled trial to address this matter would be of interest.

Although the incidence of major complications was low and comparable to the general population, cases of PV stenosis, most of them asymptomatic, have been noted (ranging from 3\% to $4.8 \%$ ). In two reports these occurred in the setting of nonostial PV isolation..$^{15} 25$ As pulmonary venogram was not routinely performed in all cases we cannot report on the prevalence of this complication and this reflects the Registry data in the general $\mathrm{AF}$ ablation populations as asymptomatic PV stenosis is not reported routinely. ${ }^{44}$

Given the small numbers of patients in all included studies, it is unclear if the apparently high rate of PV stenosis truly reflects a higher risk in this population or if it is a product of small sample sizes in the reporting studies. ${ }^{15}$ It has been suggested by Kilicaslan $e t a l^{15}$ that patients with HCM might be prone to more exaggerated hypertrophic tissue responses leading to tissue stenosis. This is yet to be confirmed, but it may also be a contributory factor for more frequent gap formation and PV reconnection in the HCM population. The possible increase in PV stenosis in this subset of patients warrants clarification, and the electrophysiologist performing cases in these patients should be aware of this potential complication and try to deliver lesions as far away as possible from the PVs.

Two systematic reviews on the role of catheter ablation of AF in patients with HCM have been recently published. ${ }^{45} 46$ However, unlike these, where the overall success rate of the procedure is reported, ours is the first meta-analysis with a casecontrol design. This is of importance, as it is the first paper allowing comparisons between patients with HCM versus other patients undergoing $\mathrm{AF}$ ablation, providing a better understanding of the true effectiveness of the catheter ablation in this setting. As included studies in the aforementioned systematic reviews ${ }^{45} 46$ span for almost a decade, simply pooling the success rates in those cohorts of patients with HCM without having any control group/comparator, makes the pooled OR impossible to interpret.

\section{LIMITATIONS}

There are some limitations to this meta-analysis. First, there is a paucity of data and studies allowing the comparison of patients with HCM and patients without HCM. As a result of this (small number of included studies and patients) this analysis has low power. However, these data are able to demonstrate differences in outcomes of catheter ablation of AF in patients with $\mathrm{HCM}$ and patients without HCM. Second, the ratio of patients with HCM to controls differs across studies. Third, moderate to high heterogeneity was observed across the included studies. A careful analysis of figures 2 and 3, shows that the rate of relapse and number of redo procedures in patients with HCM stands out as higher in the cohort published by McCready et al. ${ }^{19}$ This can be attributable to the fact that all patients in that study had persistent AF, and in most circumstances this was long-standing persistent. Lastly, data quality was modest, with no data derived from randomised controlled trials or large registries. The abovementioned factors suggest that the reliability of the estimated effect sizes may be suboptimal.

\section{CONCLUSIONS}

Data regarding catheter ablation of AF in the HCM population are scarce and of modest quality. The observed complication rate was low. Although outcomes seem less favourable than for the general population, with a twofold higher risk of relapse, more frequent need of repeat procedures and concomitant use of antiarrhythmic drugs, ablation can be a valuable option for symptomatic drug-refractory patients with HCM, particularly in those with paroxysmal $\mathrm{AF}$ and smaller atria.

\section{Key messages}

What is already known about this subject?

- Atrial fibrillation is a common finding in patients with hypertrophic cardiomyopathy, and anti-arrhythmic drugs are frequently not effective enough for a rhythm control strategy.

\section{What does this study add?}

- This meta-analysis confirms that catheter ablation can be a valuable option in patients with hypertrophic cardiomyopathy and atrial fibrillation.

- However, the overall success rate of an atrial fibrillation ablation procedure in patients with hypertrophic cardiomyopathy is worse than for the general population. Best candidates are patients with small atria and paroxysmal AF.

\section{How might this impact on clinical practice?}

- These results reinforce the role of appropriate patient selection and ideal timing of the procedure. Referral in early states of disease progression may optimize the chances of an effective rhythm control strategy.

Contributors RP and PDL planned this meta-analysis population, intervention, comparison and outcome (PICO) approach. RP and KP were responsible for data collection. GB, NS, KB and NP confirmed data collection, study selection criteria and performed study quality assessment. JM provided patient level data for one of the studies. RP performed the statistical analysis and wrote the first draft of the paper, which was thoroughly revised by PDL and PE, who provided important critical input. A new and revised version of the paper was prepared and sent to all authors, who provided suggestions and approval after the final version of the paper was written.

Funding Research supported by University College of London Hospitals Biomedicine Research Centre, a Partnership between University College of London and University College of London Hospitals NHS Trust, funded by the National Institute for Health Research (NIHR); British Heart Foundation.

Competing interests RP has received training grant from Boston Scientific, and Sorin Group and a Research Grant from Medtronic. PDL has research grants and speaker fees from Boston Scientific, St Jude, Research Grants from Medtronic and Biotronik, Funding from UCLH Biomedicine NIHR.

Provenance and peer review Not commissioned; externally peer reviewed.

\section{REFERENCES}

1 Maron BJ, Gardin JM, Flack JM, et al. Prevalence of hypertrophic cardiomyopathy in a general population of young adults. Echocardiographic analysis of 4111 subjects in the CARDIA Study. Coronary Artery Risk Development in (Young) Adults. Circulation 1995;92:785-9. 
2 Guttmann OP, Rahman MS, O'Mahony C, et al. Atrial fibrillation and thromboembolism in patients with hypertrophic cardiomyopathy: systematic review. Heart 2014;100:465-72.

3 Olivotto I, Cecchi F, Casey SA, et al. Impact of atrial fibrillation on the clinical course of hypertrophic cardiomyopathy. Circulation 2001;104:2517-24.

4 January CT, Wann LS, Alpert JS, et al. 2014 AHA/ACC/HRS guideline for the management of patients with atrial fibrillation: a report of the American College of Cardiology/American Heart Association Task Force on Practice Guidelines and the Heart Rhythm Society. J Am Coll Cardiol 2014;64:e1-76.

5 Robinson K, Frenneaux MP, Stockins B, et al. Atrial fibrillation in hypertrophic cardiomyopathy: a longitudinal study. J Am Coll Cardiol 1990;15:1279-85.

6 McKenna WJ, Harris L, Rowland $E$, et al. Amiodarone for long-term management of patients with hypertrophic cardiomyopathy. Am J Cardiol 1984;54:802-10.

7 Tendera M, Wycisk A, Schneeweiss A, et al. Effect of sotalol on arrhythmias and exercise tolerance in patients with hypertrophic cardiomyopathy. Cardiology 1993;82:335-42.

8 Calkins H, Kuck KH, Cappato R, et al. 2012 HRS/EHRA/ECAS expert consensus statement on catheter and surgical ablation of atrial fibrillation: recommendation for patient selection, procedural techniques, patient management and follow-up, definitions, endpoints, and research trial design: a report of the Heart Rhythm Society (HRS) Task Force on Catheter and Surgical Ablation of Atrial Fibrillation. Developed in partnership with the European Heart Rhythm Association (EHRA), a registered branch of the European Society of Cardiology (ESC) and the European Cardiac Arrhythmia Society (ECAS); and in collaboration with the American College of Cardiology (ACC), American Heart Association (AHA), the Asia Pacific Heart Rhythm Society (APHRS), and the Society of Thoracic Surgeons (STS). Endorsed by the governing bodies of the American College of Cardiology Foundation, the American Heart Association, the European Cardiac Arrhythmia Society, the European Heart Rhythm Association, the Society of Thoracic Surgeons, the Asia Pacific Heart Rhythm Society, and the Heart Rhythm Society. Heart Rhythm 2012;9:632-696.e21.

9 Menzies D. Systematic reviews and meta-analyses. Int J Tuberc Lung Dis 2011;15:582-93.

10 Wells G, Shea B, O'Connell D, et al. The Newcastle-Ottawa Scale (NOS) for assessing the quality of nonrandomised studies in meta-analyses. http://www.ohri. ca/programs/clinical_epidemiology/oxford.htm (accessed 11 July 2015).

11 Moher D, Liberati A, Tetzlaff J, et al. PRISMA Group. Preferred reporting items for systematic reviews and meta-analyses: the PRISMA statement. Ann Intern Med 2009;151:264-9.

12 Higgins JP, Thompson SG, Deeks JJ, et al. Measuring inconsistency in meta-analyses. BMJ 2003;327:557-60.

13 Higgins JPT, Green S, eds. Cochrane Handbook for Systematic Reviews of Interventions Version 5.1.0 (updated March 2011). The Cochrane Collaboration, 2011. http://www.cochrane-handbook.org

14 Liu X, Ouyang F, Mavrakis $\mathrm{H}$, et al. Complete pulmonary vein isolation guided by three-dimensional electroanatomical mapping for the treatment of paroxysmal atria fibrillation in patients with hypertrophic obstructive cardiomyopathy. Europace 2005:7:421-7.

15 Kilicaslan F, Verma A, Saad E, et al. Efficacy of catheter ablation of atrial fibrillation in patients with hypertrophic obstructive cardiomyopathy. Heart Rhythm 2006;3:275-80.

16 Gaita F, Di Donna P, Olivotto I, et al. Usefulness and safety of transcatheter ablation of atrial fibrillation in patients with hypertrophic cardiomyopathy. Am J Cardiol 2007;99:1575-81

17 Bunch TJ, Munger TM, Friedman PA, et al. Substrate and procedural predictors of outcomes after catheter ablation for atrial fibrillation in patients with hypertrophic cardiomyopathy. J Cardiovasc Electrophysiol 2008;19:1009-14.

18 Di Donna P, Olivotto I, Delcrè SD, et al. Efficacy of catheter ablation for atrial fibrillation in hypertrophic cardiomyopathy: impact of age, atrial remodelling, and disease progression. Europace 2010;12:347-55.

19 McCready JW, Smedley T, Lambiase PD, et al. Predictors of recurrence following radiofrequency ablation for persistent atrial fibrillation. Europace 2011;13:355-61.

20 Derejko P, Polańska M, Chojnowska L, et al. Catheter ablation of atrial fibrillation in patients with hypertrophic cardiomyopathy: atrial fibrillation type determines the success rate. Kardiol Pol 2013;71:17-24.

21 Santangeli P, Di Biase L, Themistoclakis S, et al. Catheter ablation of atrial fibrillation in hypertrophic cardiomyopathy: long-term outcomes and mechanisms of arrhythmia recurrence. Circ Arrhythm Electrophysiol 2013;6:1089-94.

22 Yan Q, Dong JZ, Long DY, et al. Catheter ablation of atrial fibrillation in patients with hypertrophic cardiomyopathy. Eur Heart J 2013;34:S102.

23 Hayashi $H$, Hayashi M, Miyauchi $Y$, et al. Left atrial wall thickness and outcomes of catheter ablation for atrial fibrillation in patients with hypertrophic cardiomyopathy. J Interv Card Electrophysiol 2014;40:153-60.

24 Contreras-Valdes FM, Buxton AE, Josephson ME, et al. Atrial fibrillation ablation in patients with hypertrophic cardiomyopathy: long-term outcomes and clinical predictors. J Am Coll Cardiol 2015;65:1485-7.
25 Müssigbrodt A, Kosiuk J, Koutalas E, et al. Results of catheter ablation of atrial fibrillation in hypertrophied hearts - comparison between primary and secondary hypertrophy. J Cardiol 2015;65:474-8.

26 Okamatsu $H$, Ohara T, Kanzaki $H$, et al. Impact of left ventricular diastolic dysfunction on outcome of catheter ablation for atrial fibrillation in patients with hypertrophic cardiomyopathy. Circ J 2015;79:419-24.

27 Wen SN, Liu N, Li SN, et al. QTc Interval Prolongation Predicts Arrhythmia Recurrence After Catheter Ablation of Atrial Fibrillation in Patients With Hypertrophic Cardiomyopathy. Circ J 2015;79:1024-30.

28 Klues HG, Schiffers A, Maron BJ. Phenotypic spectrum and patterns of left ventricular hypertrophy in hypertrophic cardiomyopathy: morphologic observations and significance as assessed by two-dimensional echocardiography in 600 patients. J Am Coll Cardiol 1995;26:1699-708.

29 Gersh BJ, Maron BJ, Bonow RO, et al. 2011 ACCF/AHA Guideline for the Diagnosis and Treatment of Hypertrophic Cardiomyopathy: a report of the American College of Cardiology Foundation/American Heart Association Task Force on Practice Guidelines. Developed in collaboration with the American Association for Thoracic Surgery, American Society of Echocardiography, American Society of Nuclear Cardiology, Heart Failure Society of America, Heart Rhythm Society, Society for Cardiovascular Angiography and Interventions, and Society of Thoracic Surgeons. J Am Coll Cardiol 2011;58:e212-60.

30 McKenna WJ, Spirito P, Desnos M, et al. Experience from clinical genetics in hypertrophic cardiomyopathy: proposal for new diagnostic criteria in adult members of affected families. Heart 1997;77:130-2.

31 Maron BJ, Maron MS. Hypertrophic cardiomyopathy. Lancet 2013;381: 242-55.

32 Maron BJ, Pelliccia A, Spirito P. Cardiac disease in young trained athletes. Insights into methods for distinguishing athlete's heart from structural heart disease, with particular emphasis on hypertrophic cardiomyopathy. Circulation 1995:91:1596-601.

33 Ravelli F, Allessie M. Effects of atrial dilatation on refractory period and vulnerability to atrial fibrillation in the isolated Langendorff-perfused rabbit heart. Circulation 1997:96:1686-95.

34 Maron BJ, McKenna WJ, Danielson GK, et al. American College of Cardiology/ European Society of Cardiology clinical expert consensus document on hypertrophic cardiomyopathy. A report of the American College of Cardiology Foundation Task Force on Clinical Expert Consensus Documents and the European Society of Cardiology Committee for Practice Guidelines. J Am Coll Cardiol 2003:42:1687-713.

35 Satoh T, Zipes DP. Unequal atrial stretch in dogs increases dispersion of refractoriness conducive to developing atrial fibrillation. J Cardiovasc Electrophysiol 1996; 7:833-42

36 Ohtani K, Yutani C, Nagata $S$, et al. High prevalence of atrial fibrosis in patients with dilated cardiomyopathy. J Am Coll Cardiol 1995;25:1162-9.

37 Verheule S, Sato T, Everett TT, et al. Increased vulnerability to atrial fibrillation in transgenic mice with selective atrial fibrosis caused by overexpression of TGF-beta1. Circ Res 2004;94:1458-65

38 Gruver EJ, Fatkin D, Dodds GA, et al. Familial hypertrophic cardiomyopathy and atrial fibrillation caused by Arg663His beta-cardiac myosin heavy chainmutation. Am J Cardiol 1999;83(Suppl 12A):13H-8.

39 Ogimoto A, Hamada M, Nakura J, et al. Relation between angiotensin-converting enzyme II genotype and atrial fibrillation in Japanese patients with hypertrophic cardiomyopathy. J Hum Genet 2002;47:184-9.

40 Lan $F$, Lee AS, Liang $P$, et al. Abnormal calcium handling properties underlie familia hypertrophic cardiomyopathy pathology in patient-specific induced pluripotent stem cells. Cell Stem Cell 2013;12:101-13.

41 Cannon 3rd RO, Rosing DR, MaronB J, et al. Myocardial ischemia in patients with hypertrophic cardiomyopathy: contribution of inadequate vasodilator reserve and elevated left ventricular filling pressures. Circulation 1985;71: 234-43.

42 Schafers M, Dutka D, Rhodes CG, et al. Myocardial presynaptic and postsynaptic autonomic dysfunction in hypertrophic cardiomyopathy. Circ Res 1998:82:57-62.

43 Providência R, Lambiase PD, Srinivasan N, et al. Is There Still a Role for CFAE Ablation in Addition to Pulmonary Vein Isolation in Patients with Paroxysmal and Persistent Atrial Fibrillation? A Meta-Analysis of 1,415 Patients. Circ Arrhythm Electrophysiol 2015;8:1017-29.

44 Cappato R, Calkins H, Chen SA, et al. Worldwide survey on the methods, efficacy, and safety of catheter ablation for human atrial fibrillation. Circulation 2005;111:1100-15.

45 Zhao DS, Shen Y, Zhang Q, et al. Outcomes of catheter ablation of atrial fibrillation in patients with hypertrophic cardiomyopathy: a systematic review and meta-analysis. Europace 2015. doi:10.1093/europace/euv339

46 Ha HS, Wang N, Wong S, et al. Catheter ablation for atrial fibrillation in hypertrophic cardiomyopathy patients: a systematic review. J Interv Card Electrophysiol 2015;44:161-70. 